\title{
The search for extrasolar planets with BRITE
}

\author{
R. Dvorak ${ }^{1}$, Á. Bazsó ${ }^{1}$ \\ ${ }^{1}$ Institut für Astronomie, Türkenschanzstrasse 17, 1180 Vienna, Austria
}

\begin{abstract}
We summarize in this report the possibilities of observing the reflecting light of a close by giant planet. BRITE could do these kind of observations only for very bright stars, but the chances that such a bright star with a close by large planet, a hot Jupiter, exists in the Solar environment are rather small. We discuss our present knowledge of analyzing reflecting light in light curves of stars.
\end{abstract}

\section{Introduction}

Since more than ten years an increasing number of extrasolar planets (exoplanets) around main sequence stars has been detected. By November 2007264 planets are known in 215 extrasolar planetary systems (EPS) ${ }^{1}$. The predominant detection method is by radial-velocity measurement of the Doppler-shift in the spectrum of the star, an indirect method.

For BRITE, which will make photometric observations, light-curves and their interpretation, it is necessary to develop tools and methods to study them, and to have working models for a great class of possible observations.

Several problems arise for the direct detection and imaging of an exoplanet, among them (i) the problem of angular separation of the planet from the star, and (ii) the planet/star flux ratio are the main limiting factors. Take for instance a main sequence star of type $\mathrm{G} 2 \mathrm{~V}$ at a distance of $10 \mathrm{pc}$ and a planet with semi-major axis of $a=0.4 \mathrm{AU}$, then the angular separation is as low as 0.4 arcseconds, and the planet/star flux ratio at optical wavelengths is below $10^{-9}$.

The reflection off an exoplanet's surface cannot be modeled by assuming simple reflection models or by extrapolating solar-system data. One has to consider the planet's orbital distance due to effects on its composition, as well as the constituents of its atmosphere, also the particles and their sizes play an important role.

\footnotetext{
${ }^{1}$ according to the internet site (exoplanet.eu) provided by Jean Schneider from the Paris observatory
} 
We plan to give an overview of the different effects acting on the lightcurves of exoplanets, as described by Sudarsky et al. (2005), whom we follow closely in this article. For further literature we refer to Hubbard et al. (2001), Burrows et al. (2004), Rowe et al. (2006) and also Burrows et al. (2007).

We consider only Jupiter-type giant planets-from now on referred to as extrasolar giant planets (EGP) - with one Jupiter mass $\left(1 M_{J}\right)$ and an age of 5 Gyrs (neglecting evolutionary effects). Furthermore the planet is assumed to be at orbital distances ranging from 0.2 to $15 \mathrm{AU}$ (in order that the angular separation of the planet from the star is possible), and it orbits a main-sequence star of type G2V with solar metallicity.

First we give an overview of the necessary notions, then we describe the variation of the light-curve for different orbital elements. Following that the form of the light-curve at different wavelengths will be investigated, as well as the impact of clouds and small particles in the atmosphere.

\section{Basic notions}

The phase angle $\alpha$ is the angle in the plane observer-star-planet, whose vertex lies at the planet. This angle determines the brightness of an EGP, since the planet/star flux ratio

$$
F_{P} / F_{S}=A_{g}\left(\frac{r}{a}\right)^{2} \phi(\alpha)
$$

depends on it via the phase function $\phi(\alpha)=E(\alpha) / E(0)$, where $r$ is the planet's radius, $a$ its orbital distance (semi-major axis), and

$$
A_{g}=\frac{E(0) d^{2}}{\pi r^{2} S}
$$

the geometrical albedo (reflectivity of the planet at full phase $\alpha=0$ compared to the incident flux $S$ from the star onto a perfect disc of the same radius $r)$.

For a light-curve over one orbital period of the planet one must solve the planet/star flux ratio for both $A_{g}$ and $\phi(\alpha)$, which contain the function $E(\alpha)$ the energy per second per unit area per unit solid angle received at phase $\alpha$-which itself contains an integral over the reflection coefficient $\rho$ that is depending on many parameters (e.g. temperature, pressure, wavelength, etc.). If we need the spherical albedo $A_{s}=q A_{g}$ one has to calculate the phase integral

$$
q=2 \int_{0}^{\pi} \mathrm{d} \alpha \phi(\alpha) \sin \alpha
$$




\section{Variation of the Orbital Elements}

The relation of the phase angle to the orbital elements is given by

$$
\cos \alpha=\sin (\varphi+\omega) \sin (i) \sin (\Omega)-\cos (\Omega) \cos (\varphi+\omega)
$$

where $\varphi$ is the planet's orbital angle (or "true anomaly"), $i$ the orbital inclination, $\omega$ the argument of periastron, and $\Omega$ the longitude of the ascending node.

By default Sudarsky et al. chose a highly inclined orbit of $i \geq 80^{\circ}\left(i=0^{\circ}\right.$ means face-on, and $i=90^{\circ}$ is edge-on), and $\Omega=90^{\circ}$.

Most of the discovered exoplanets have non-vanishing eccentricities, and there are even some planets in highly eccentric orbits. In contrast to a lightcurve for zero eccentricity $(e=0)$, the light-curves differ for an eccentric orbit, the symmetric shape is distorted to a sharp peak around the pericenter position. The maximum of the planet/star flux ratio does not appear for fullphase $(\alpha=0)$, as in the circular case. For a high eccentricity the planet appears to be brighter, due to the higher incident stellar flux, for $e=0.6$ the planet appears three times brighter compared to $e=0$.

Variation of the orbital inclination does affect the flux ratio, it is highest for nearly edge-on orbits, and the peak value is approximately three times higher than for face-on orbits. It is remarkable that for $i=0$ and non-vanishing eccentricity there is still a variation in the flux ratio, because the planet's distance to the star changes.

The form of the light-curve and the position of the maximum for an elliptical orbit is shifted with varying $\Omega$. The optimal value for this parameter would be $\Omega=90^{\circ}$, for then the line of nodes would be perpendicular to the line of sight.

The value of the argument of periastron $\omega$ plays an important role for the maximum of the light-curve, since for $\omega=90^{\circ}$ and not edge-on orientation the opposition of the planet takes place in the perihelion when its distance from the star is smallest, for $\omega=270^{\circ}$ it is exactly the other way round.

\section{Light-curves at different wavelengths}

For a fixed value of $i=80^{\circ}$ and circular orbits Sudarsky et al. (2005) investigated the change in the light-curve for different values of the semi-major axis and for different wavelengths. They included the formation of clouds by condensation if the atmospheric temperature drops below a certain value. They found that especially water and ammonia clouds play important roles for EGPs that are farther out than $2 \mathrm{AU}$, while for EGPs nearer than $1 \mathrm{AU}$ these clouds cannot form, and all atmospheric constituents remain in gaseous form. 


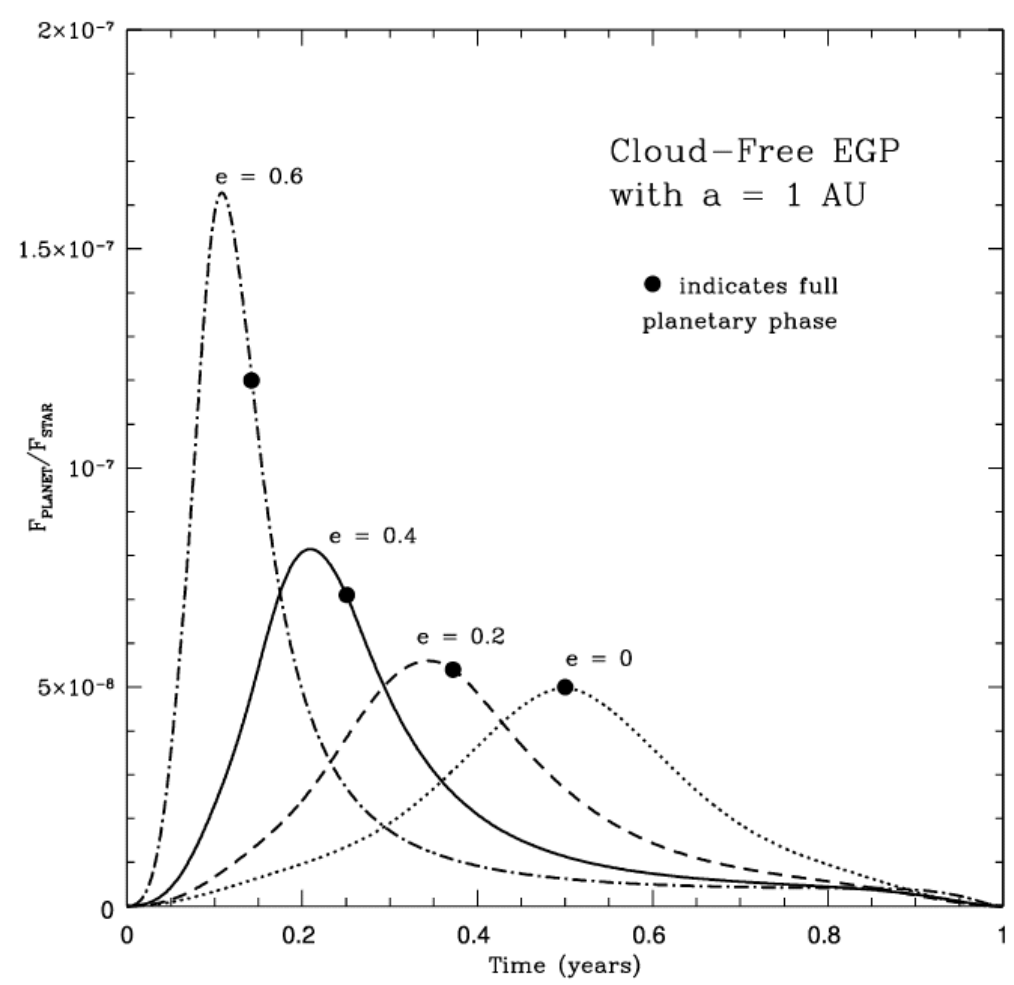

Figure 1: Variation of the light-curve for different values of the eccentricity (after Sudarsky et al. (2005), Figure 17).

In the simplest case the planet/star flux ratio would follow the $1 / a^{2}$ law, that means that the flux ratio decreases with increasing orbital distance. In the models the behavior is different from that, the flux ratio is governed by different wavelengths at different distances. For close-in EGPs the near-infrared flux at $\lambda=1.25 \mu \mathrm{m}$ dominates with a flux ratio of approximately $10^{-6}$, partly due to thermal re-emission of the starlight, so that according to this the search of near but separable exoplanets should concentrate to the IR.

At a distance of $1 \mathrm{AU}$ there is a change and the visible wavelengths become and remain predominant for all considered values up to $a=15 \mathrm{AU}$. Near EGPs around $1 \mathrm{AU}$ are more luminous at $\lambda=0.55 \mu \mathrm{m}$ than in the near infrared. In total there is a factor of nearly five between $\lambda=0.55 \mu \mathrm{m}$ and $\lambda=1.0 \mu \mathrm{m}$ for $1 \mathrm{AU}$; while this gap is smaller for a more distant EGP. With increasing 


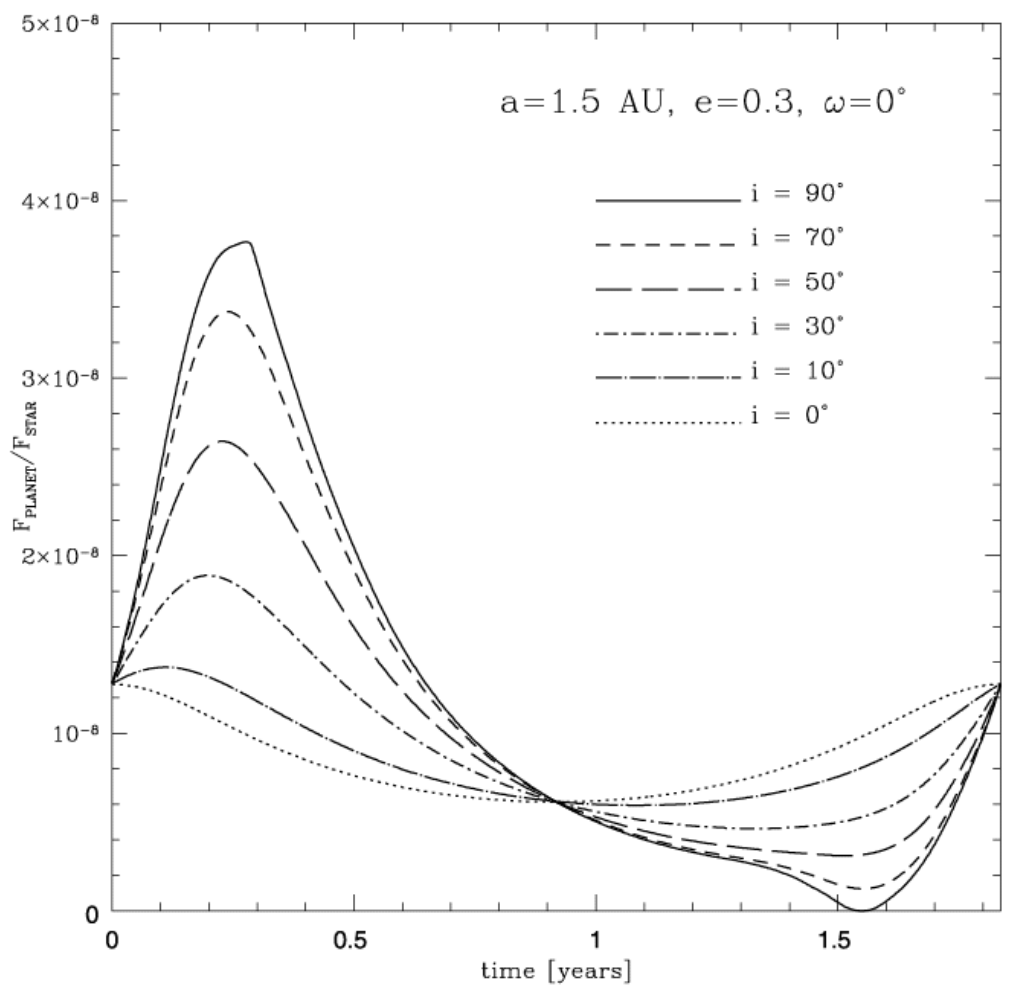

Figure 2: Variation of the light-curve for different orbital inclinations (after Sudarsky et al. (2005), Figure 20).

distance from the star the atmosphere of an EGP can undergo a change, and the formation of clouds-at first only water ice clouds in the upper atmospherebrightens the EGP in the optical region. An EGP at 2 AU shows a planet/star flux that is about $50 \%$ of the corresponding flux for $\lambda=0.55 \mu \mathrm{m}$ of the $1 \mathrm{AU}$ planet, this is more than to expect for it without the effect of the clouds.

For even higher distances beyond $a>6 \mathrm{AU}$ still the smaller wavelengths around $\lambda=0.55 \mu \mathrm{m}$ dominate, and eventually ammonia ice clouds can form, with a deeper water ice cloud. The maximum planet/star flux is below $10^{-9}$ for $\lambda=0.55 \mu \mathrm{m}$, and can be as low as $10^{-10}$ for very distant planets $(a=10 \mathrm{AU})$ in the IR $(\lambda=1.0 \mu m)$. In general one has to expect that the IR-brightness is only about $20-40 \%$ of the optical brightness. 


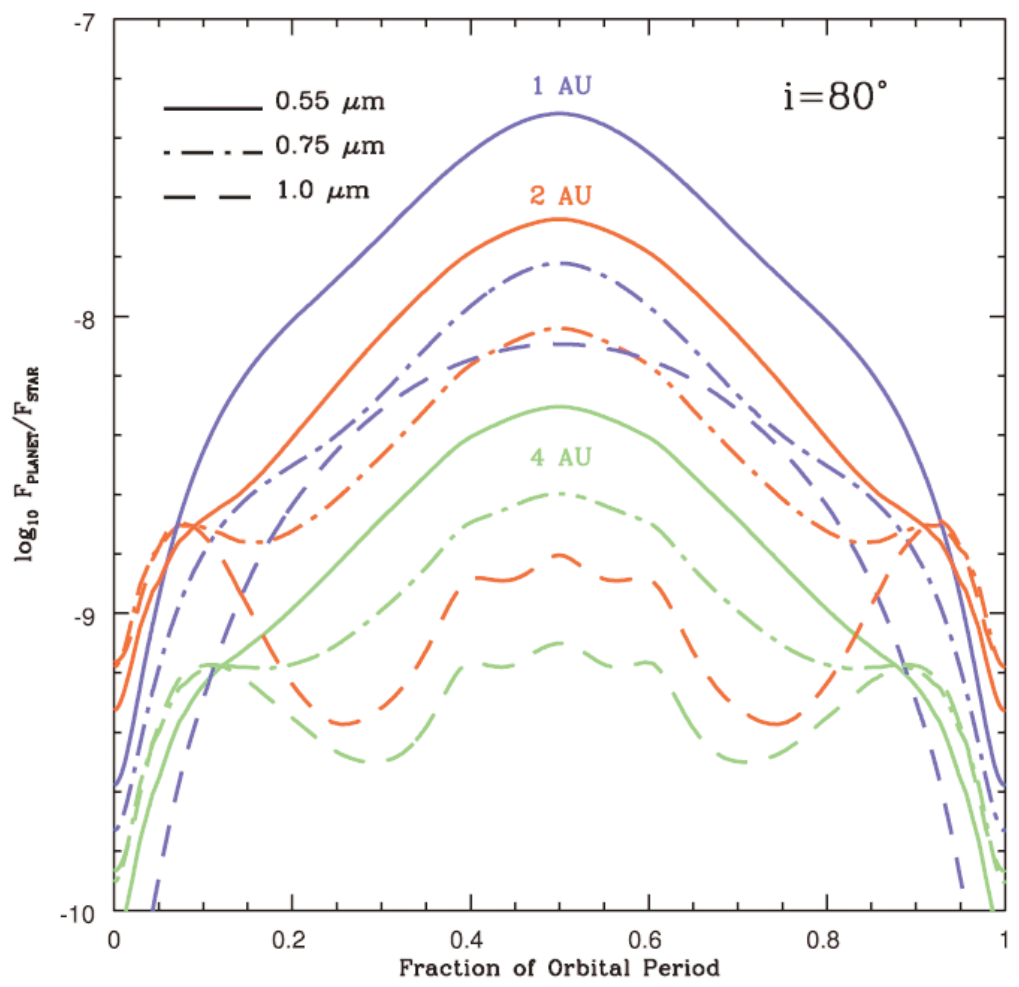

Figure 3: Variation of the light-curve for planets with different orbital distances, the dependence of the light-curve on the wavelength is also included (after Sudarsky et al. (2005), Figure 10).

\section{Effect of particles and clouds}

An EGP having clouds in its atmosphere is brighter in the optical spectral region, because of reflective Rayleigh scattering, an effect we also encounter in our Solar System.

Sometimes clouds can be a transient phenomena, provided that the EGP is on a highly eccentric orbit, it is possible that clouds form and dissolve whenever the EGP leaves the vicinity of the star or approaches it, respectively. The type of clouds (water, ammonia) plays an important role, too, as described above.

Grains and ice particles of different sizes can form at suitably low temperatures, but not at orbital distances below $2 \mathrm{AU}$, as found in the paper. From 


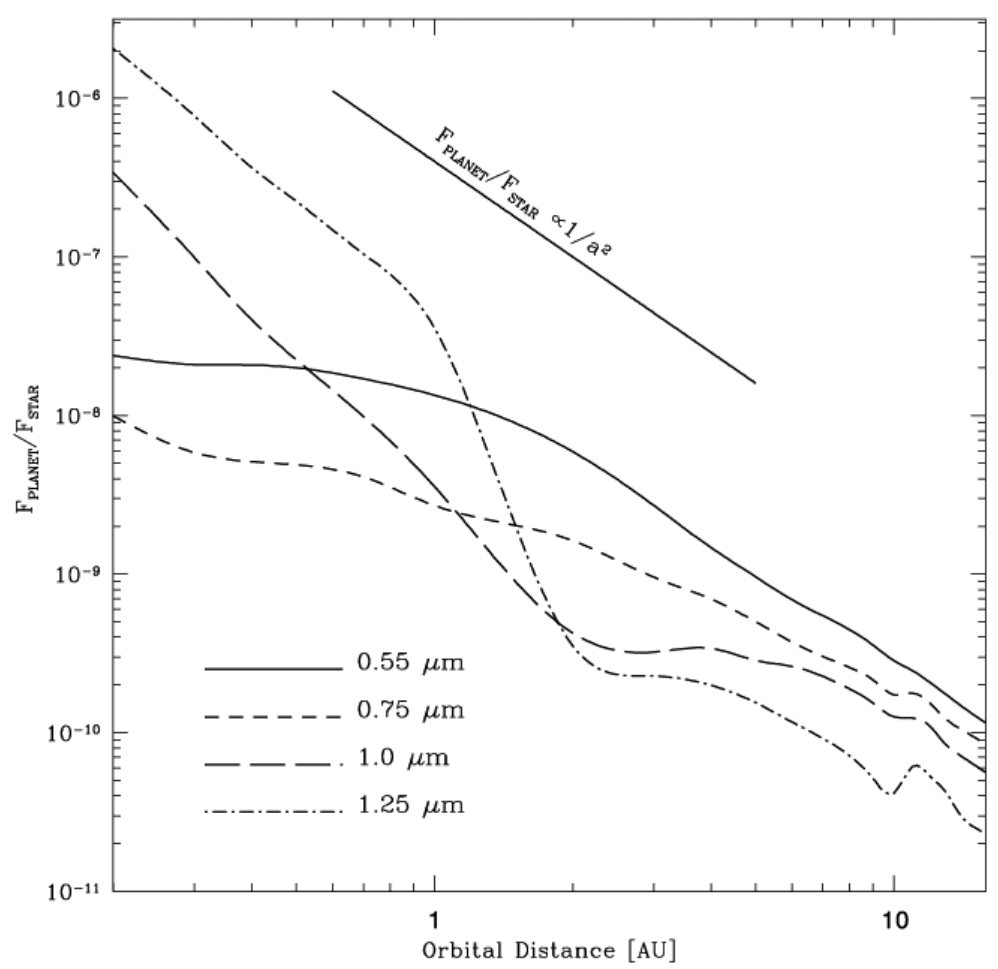

Figure 4: At different orbital distances the planet/star flux ratio is dominated by the near-IR wavelengths for close-in planets, while the flux in the visible region is increased by reflection and scattering for distances greater than 1 AU (after Sudarsky et al. (2005), Figure 12).

the viewpoint of scattering the smallest particles with diameters of about $1 \mu \mathrm{m}$ have the strongest effect on the light-curve, the planet/star flux ration is increased by a factor of 2-4 compared to a cloudless planet (for $\lambda$ in the range $0.55-0.75 \mu \mathrm{m})$, bigger particles up to $100 \mu \mathrm{m}$ diameter have less effect.

\section{Summary and Conclusions}

The main conclusions are the following

- The phase function of an EGP is strongly wavelength dependent, as well as the (geometrical or spherical) albedo and the phase integral, that rely 


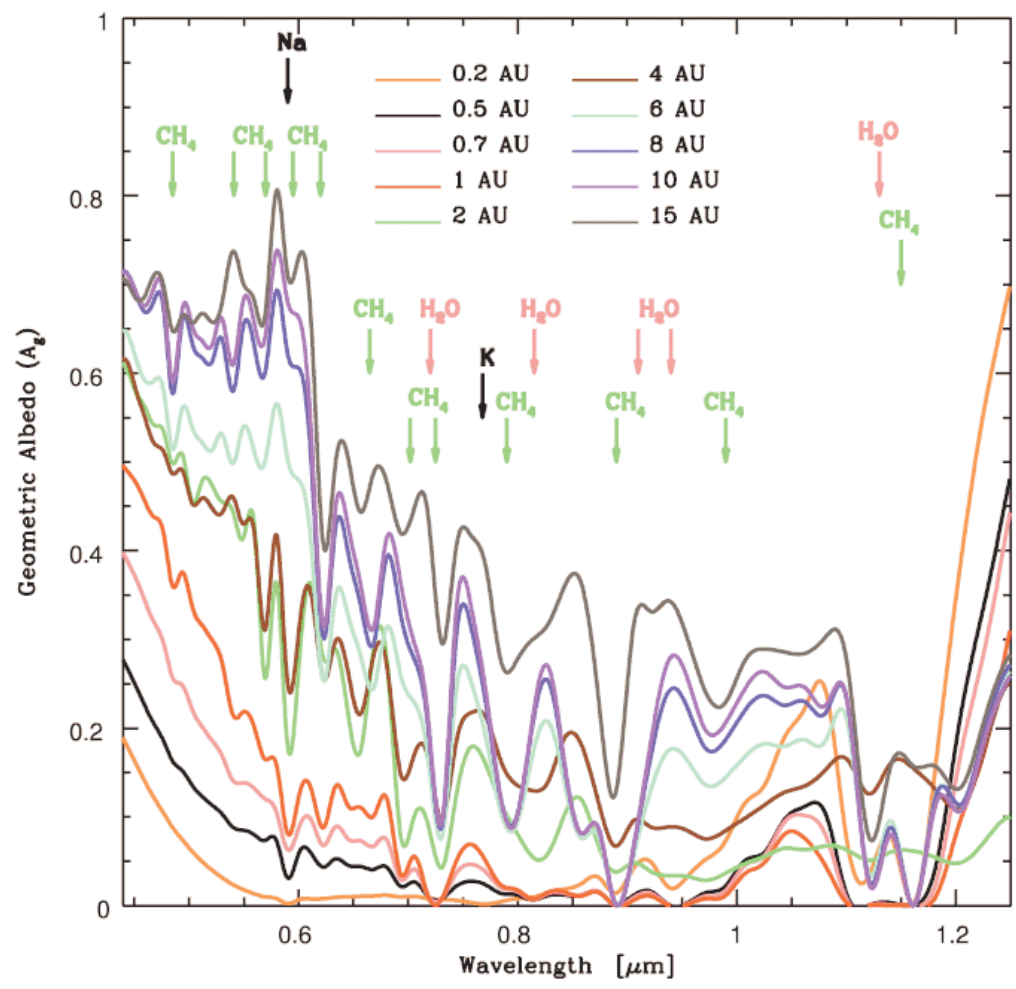

Figure 5: Geometric albedo in the region considered for wavelengths between $0.55 \mu \mathrm{m}$ and $1.25 \mu \mathrm{m}$. The maximum planet/star flux is below $10^{-9}$ for $\lambda=0.55 \mu \mathrm{m}$, and can be as low as $10^{-10}$ for very distant planets $(a=10 \mathrm{AU})$ in the $\operatorname{IR}(\lambda=1.0 \mu \mathrm{m})$. In general one has to expect that the IR-brightness is only about $20-40 \%$ of the optical brightness. (after Sudarsky et al. (2005), Figure 9).

on it. EGPs like those considered reflect more light in the optical than in the near infrared.

- Clouds consisting of small particles increase the planet/star flux ratio relative to that of EGPs with bigger particles or cloudless EGPs.

- In elliptical orbits EGPs exhibit higher flux ratios than for circular orbits, and the maximum of the light-curve does not coincide with full phase. Additionally for strongly eccentric orbits the atmospheric composition might change periodically, having an effect on the light-curve. 
- EGPs with highly inclined orbits are generally brighter at full phase, and they should be best visible at full phase rather than at their greatest elongation, provided that the planet's orbit is not fully edge-on.

- For BRITE it will be difficult to observe a bright star surrounded by a close Gasgiant, because the number of bright stars in the Sun's vicinity with such a planet is - as for as we can estimate - quite small.

\section{References}

Burrows, A., Budaj, J., Hubeny, I. 2007, astro-ph 0709.4080

Burrows, A., Sudarsky, D., Hubeny, I. 2004, ApJ, 609, 407

Hubbard, W.B., Fortney, J.J.,Lunine, J.I., et al. 2001, ApJ, 560, 413

Rowe, J.F., Matthews, J.M., Seager, S. et al. 2006, ApJ, 646, 1241

Sudarsky, D.,Burrows, A., Hubeny, I., et al. 2005, ApJ, 627, 520

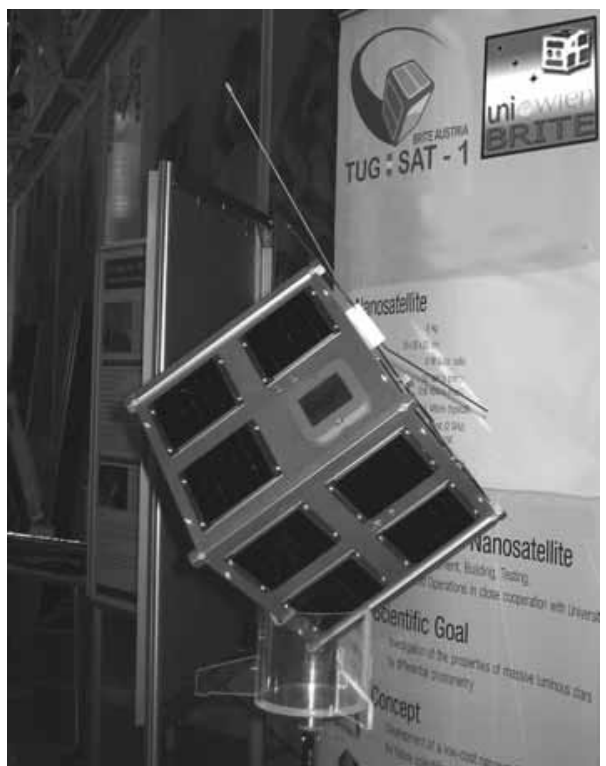

The BRITE nanosatellite model. 